\title{
Distance Education An Opportunity to Support Nursing in Southeast Asia
}

\author{
Robert L Anders ${ }^{1}$, Wipada Kunaviktikul ${ }^{2}$ \\ ${ }^{1}$ University of Texas at El Paso, Delta, United States \\ ${ }^{2}$ Chiang Mai University, Chiang Mai, Thailand
}

Aims and Objectives: This discursive paper aims to discuss the potential creation of a bridging BNS program in selected Southeast Asian countries to increase the number of nurse educators for the goal of having local educators to increase the number of nurses in their respective countries.

Background: Nursing in Thailand has evolved from its original support by the past King and Queen of Thailand. Now, most nurses have a BNS degree for entry into practice. The Chiang Mai University has succeeded in delivering nursing and training programs in Association of Southeast Asian Nations (ASEAN).

Design: A discursive article is describing a distance educational model.

Methods: Literature from nursing education in Thailand, World Health Organization (WHO) projected shortage of nurses, nursing education reform, and distance education nursing programs informed this article's opinions. The use of distance education bridging BNS programs has the potential to build faculty capacity quickly.

Conclusions: Thailand can educate, to some extent, through distance education, international nurses within the ASEAN region. Providing baccalaureate education will increase the capacity of many of these countries to educate their nurses.

Relevance to Clinical Practice: Creating opportunities for preparing baccalaureate nurse educators for low-income countries within South East Asia supports improving health outcomes.

Keywords: ASEAN; International nursing; Nursing education; Nursing policy; Overseas study; Thailand

\section{INTRODUCTION}

This discursive paper aims to discuss the potential creation of a nursing baccalaureate bridge program in selected Southeast Asian countries to increase the number of nurse educators, thereby increasing the number of graduates, to address the nursing shortage in those countries.

\section{BACKGROUND}

A strategic response to the needs of Asian populations should begin with a feasibility study on how best to develop leaders who can assume responsibility for curriculum review and renewal in their own countries. Thailand has the experience to take the lead in a curriculum development initiative that builds new education programs in developing countries. There should be careful consideration given to the type of educational programs that will lead to graduate outcomes. Professional attributes should include inquiry processes, critical thinking, sound therapeutic practices, ethical-legal standards, and information literacy that enables an evidence-base for practice.

(C) Copyright 2021 International Society for Problem-Based Learning

(c) This is an Open Access article distributed under the terms of the Creative Commons Attribution Non-Commercial License (http://creativecommons.org/licenses/ by-nc/4.0/) which permits unrestricted non-commercial use, distribution, and reproduction in any medium, provided the original work is properly cited. 
In Thailand, nursing has a long tradition. In 1896 Thailand's established its first nursing school. Nursing was first introduced to Thailand by Queen Sripatchariantra, the wife of King Rama V, as a response to personal tragedy. Losing her child to cholera, coupled with the high maternal death rate at the time, compelled her to advocate for modern nursing. Given the high regard that Thais have for the royal family, royal support for nursing allowed the profession to be accepted and well respected. Required in 1978, all nursing schools offer a four-year baccalaureate program with an additional six-month midwifery certificate. The program was later revised to four years, inclusive of nursing and midwifery (Anders \& Kunaviktikul, 1999). The role of professional nurses in Thailand parallels the nursing journey found in high-income countries (Liu et al., 2015).

Currently, there are ninety-four nursing schools in Thailand, and all expect one to offer a four-year Bachelor of Nursing Science (BNS) program that includes midwifery. Of those, twenty have graduate degree programs, with eight offering doctoral programs. In Southeast Asia, Thailand has and continues to be a leader in nursing education.

Thailand is in a unique position to support and build capacity in nursing in other Southeast Asian countries and providing regional support in nursing education is a priority (Kunaviktikul et al., 2019). Both the World Health Organization (WHO) and the International Council of Nurses stress that the sustainable development goals and universal health coverage are only achievable with a professional nursing and midwifery workforce (International Council of Nurses and Nursing Now Welcome 2020 as International Year of The nurse and the Midwife, 2020). However, to reach these goals, changes are needed in nursing policy and credentialing. An educated nursing workforce is a key to increasing support for policies on nursing licensure, competencies, and credentials (Kunaviktikul \& Guptarak, 2019).

In Southeast Asia, nursing education has substantially improved through the availability of bachelor's degree bridging programs and master and doctoral degree programs (Kunaviktikul et al., 2019). Given the success in nursing education, as evidenced at Thai universities, Thailand is poised to assist the Association of Southeast Asian Nations (ASEAN) nursing leaders and clinicians in meeting sustainable development goals related to universal health coverage. Thailand has a history of providing international assistance, and nursing and midwifery capacity building for ASEAN nurse educators could be a part of Thailand's international outreach.

\section{STRUCTURAL EMPOWERMENT}

Kanter (2008) describes how workplace systems shape employee health and organizational performance. Two primary empowering frameworks are important: the structure of opportunity and the structure of control. Opportunity refers to nursing abilities to improve, learn, and develop knowledge and skills, such as earning a BNS degree. Power is the ability to mobilize information, support, and resources within the organization. Applying Kanter's structural empowerment can increase nurses' empowerment through advanced nursing education, such as achieving BNS degrees. The changes in nursing knowledge can increase positive behaviors within the nursing practice environment. Per Kanter, in theory, more empowered nurses change their practice behaviors (Kanter, 2008).

\section{NURSING IN SOUTHEAST ASIA}

Context and culture matter when the wellbeing of the population needs to be promoted and managed. Across the world, nurses offer the right skill set and attribute to respond to their people's needs. Nevertheless, more than $80 \%$ of nurses worldwide are in countries that comprise half the world's population. In 2016, the nursing shortage was around 6.6 million, and in two years, it marginally declined to 5.9 million in 2018. Approximately 5.3 million $(89 \%)$ of the deficiency are in low-and low-middle-income countries, with the number of nurses barely keeping pace with population growth. The nurse-to-population density has marginally improved, with the most massive gaps found in East Asian countries (State of the World's Nursing Report -2020, 2020).

The nursing shortage, including midwives, in low-income Southeast Asian countries, is projected to be 1.9 million (Shamila, 2020). Dr. Poonam Khetrapal Singh, WHO Director for the South-east Asia Regional Office, notes that improving education, deployment, and rural retention of nurses and midwives is a top priority. The number of nurses and midwives in the region is improving, with 3.5 million nurses and midwives equaling 18 per 10,000 people in 2018 , up from 2.9 million nurses and midwives in 2014, 16 nurses/midwives per 10,000 people (Shamila, 2020). While this progress is encouraging, more drastic changes and improvements are needed. The regional average is still far below the global average of 37 nurses per 10,000 population and the required minimum of 40 nurses per 10,000 population. By 2030, the Southeast Asia region will require an estimated 1.9 million nurses (Shamila, 2020).

Laos, Cambodia, Myanmar, and Vietnam have the most significant disparities in nursing within Southeast Asia. The WHO 
State of the World's Nursing Report - 2020 notes that there are 6,734 nurses in Laos, of which only $10 \%$ are professional nurses (baccalaureate trained). By 2030 the nursing shortage is estimated to range between 5,000 to 6,000, resulting in 9.5 nurses per 10,000 (State of the World's Nursing: Lao People Democratic Republic, 2020). There are only 6.9 nurses per 10,000 population in Cambodia, $74 \%$ of whom are professional nurses. The project nursing shortage in Cambodia ranges from 10,000 to 20,000 by 2030 (State of the World's Nursing: Cambodia, 2020). In Myanmar, there are 6.7 nurses per 10,000 population. There are 35,947 nursing professionals. The projected shortage is between 100,000 and 200,000 by 2030 (State of the World's Nursing: Myanmar, 2020). Data for Vietnam in the WHO report is mostly missing. The report indicates there are 106,654 Vietnamese nurses with no indication of the number of professional nurses. There are currently 11.4 nurses per 10,000 in Vietnam, with an estimated shortage of between 40,000 and 50,000 nurses by 2030 (State of the World's Nursing: Viet Nam, 2020).

There was a clear need for a strategic approach to the problem. The ASEAN Mutual Recognition Arrangement (MRA) on Nursing Services was adopted in 2006 to promote greater nursing cooperation between ASEAN member states. In response to the establishment ASEAN Economic Community in 2015, the Southeast and East Asian Nursing Education and Research Network (SEANERN), composed of representatives from 13 countries and regions, was founded in 2015. SEANERN's purpose is to support policy development to strengthen nursing education, clinical skills, and research capacity and promote nursing mobility across national borders. Members have reported significant variation in educational, regulatory, and clinical competencies that have restricted and continue to limit MRA policy implementation.

While the ASEAN Mutual Recognition Arrangement (MRA) on Nursing Services allows for nursing mobility across the region, which theoretically could assist with nursing shortages within the region. The essential nursing regulatory differences are challenging. All ASEAN member states have a nursing licensing examination system. Nursing education varies across ASEAN countries, and professional licensing requirements differ among ASEAN nurses. General communication and nursing examinations are typically in the country's native language. ASEAN has almost as many languages as member states. The availability of health professionals also varies; in some areas, the availability of a skilled health workforce at a regional level is above the threshold. Myanmar, Cambodia, Vietnam, and Laos fall below the WHO threshold (Efendi et al., 2018).

In theory, through the MRA, ASEAN nurses can practice in member states that are signatories to the agreement. However, to be fully operationalized is the implementation of the MRA. However, the MRA does provide a potential avenue for nurses from the ASEAN region to have access to employment and educational opportunity in member countries (Te et al., 2018).

\section{PROPOSED BSN BRIDGE PROGRAM FOR NURSE EDUCATORS IN SOUTHEAST ASIA}

Given the significant need for nurse educators, international nursing programs in the region can help address this demand. Given the advanced nursing education system in Thailand, many Faculties of Nursing can provide capacity building to nurse educators within the ASEAN. For example, the Chiang Mai University (CMU) Faculty of Nursing one of the leading nursing educational schools and offers bachelor's, master's, and doctoral degrees. CMU is known for international education and training programs throughout Asia and other regions. The China Medical Board Foundation, USA, partnered with CMU to fund graduate education for nurses from China, Laos, Myanmar, and Vietnam (Kunaviktikul et al., 2014). Armed with state-of-the-art nursing knowledge, graduates from these programs can better contribute to their home countries as nurse educators. These competencies, in turn, build the capacity of professional nurses and nursing education in these countries.

Ewha Woman's University in Seoul, Republic of Korea, successfully implemented a bachelor of nursing degree program in Cambodia. The quantitative analysis showed that the students' nursing competencies increased. Unpublished at present is a description of the program. The study provides evidence to support the effectiveness of higher education for nurses in developing countries and highlights the potential for high-quality human capital to be developed in nursing (Cha, et al., 2020).

The proposed program would be conducted in collaboration with SEANERN. It would initially explore creating a BNS bridge program with identified educational institutions within Thailand and the identified countries. Given the urgent need for additional nurse educators, nursing institutions in these countries' inquiries will occur to gauge their interest in the BNS bridge program. If there is an interest, the next step is to create a partnership between various Thailand universities, the China Medical Board, SEANERN, and identified countries.

Sought from various sources will be funding, assuming there is an interest from the targeted countries. The identified team will work with recipient countries to partner with respective Ministries of Education and Health and university leadership to seek 
in-country approval and potential funding support.

The development of BNS bridge programs for nurse educators is urgently needed. Graduates from these programs will be nurse educators prepared to contribute to their home country's nursing workforce. Graduates would then serve as faculty and facilitate the updating of nursing competencies in Southeast Asia.

\section{PROVEN STRATEGIES FOR BUILDING CAPACITY AMONG NURSING FACULTY}

The China Medical Board Foundation (created by the Rockefeller Foundation in 1914) has been a significant funder of nursing initiatives in Southeast Asia. In the last decade, the China Medical Board funded the CMU Faculty of Nursing to strengthen nursing education in Cambodia, Vietnam, Myanmar, and Laos.

The Program for Higher Education in Nursing (POHNED) was a dual master's degree program between Chiang Mai University and a Chinese university. Initial students came to CMU for intensive graduate courses. Later, faculty from CMU traveled to the partner university in China to teach classes. English was the language of instruction, including English language textbooks. Gradually, more and more instruction was handed over to the Chinese university under CMU faculty members' supervision until they could offer these graduate classes independently. Overall, the program supported nursing education in eight national medical universities in China. A total of 84 master-prepared nurses graduated from the program. POHNED is responsible in part for modernizing nursing education in China.

Chiang Mai University has demonstrated the feasibility of the model in providing high-quality short training courses and degree programs for nurses and participants from more than 50 countries trained (China Medical Board Foundation, nd). It is reasonable to assume that the same model used in collaboration with other partners will also be successful.

\section{DISTANCE EDUCATION - A COST- EFFECTIVE OPTION FOR BNS BRIDGE PROGRAMS}

The BNS bridge program would be a collaboration with selected universities with nursing programs. Chiang Mai University and other Thailand universities would provide the curriculum and classes through distance learning. Learning management platforms do not require significant bandwidth, and the internet is widely available throughout the region. Therefore, distance
BNS bridge programs can be cost-effective. One designed Thailand university will lead the partnership.

Thailand has a robust internet infrastructure. There is adequate internet connectivity on university campuses to provide a stable platform to deliver distance education. A May 2020 report on internet capacity in Southeast Asia reports the following internet penetration for countries targeted for the bridging program: Vietnam at 70.4\%; Cambodia at 47.9\%; and Laos at $42 \%$ (Internet Penetration in Southeast Asia as of May 2020, by country, 2020). All partner universities have internet availability on their respective campuses for student use in the bridge program.

Thailand university partners would provide online faculty. Each host country would provide a program coordinator, internet connection, and laptops for student use, and a local coordinator at each university to manage the program. The local coordinator would facilitate student enrollment, infrastructure support, coordination, and other help as needed. This person does not necessarily need to be a nurse. The initial training sessions for the coordinators will orient them to their responsibilities. Depending on funds, the coordinators would travel to the lead university in Thailand for orientation and training.

Moodle, an open-source learning management system, could be utilized as a distance education platform. Moodle is the world's most popular learning management system. The platform is compatible with both PC and smartphones. The system would be an ideal platform requiring little expenditure, other than training faculty members to use the platform (Moodle, n.d.). There will be separate training on using Moodle and troubleshooting technical issues with the various partner universities' information technology departments.

Students will have access to preloaded laptops with all textbooks required in the program. Backup laptops will be available to the partner university for use, as necessary. Included with the onboarding will be tutorials on how to use the laptop would be included with the onboarding of students (Lall et al., 2019).

Curriculum modification may be needed to meet the targeted countries' needs and conform to local nursing requirements. However, the integrity of the degree would still need to meet Thailand's university partner requirements. The instruction will mirror existing distance education programs at the host faculty of nursing programs. Modifying the academic assignment will be made as necessary to ensure homework and other critical thinking engagement activities reflect nursing issues in the students' country.

The evaluation protocol will follow (with modification as necessary) the existing BNS Bridge Program instrument. Included as necessary will be additional questions related to distance edu- 
cation and program support.

Teaching a generic entry into practice curriculum would be impractical, given the need for clinical faculty and skills labs. The curriculum will be the same as used at the collaborating Thailand universities. There would be only one course offered per semester year-round. Given that the students will be nurses who are employed full time, offering one class per semester should not significantly impact their workload. Through this model, an innovative international BNS bridge degree taught entirely online is possible.

The participating universities may have to create a credential assessment system to ensure that students entering the program are qualified. Successful models, particularly in US medical schools, have proven to increase matriculation and successful completion of minority students (Samuel, 2020). These programs included academic coaches to support writing, developing portfolios, test-taking strategies, and guidance on using laptops and related software programs. Additional support includes using library resources and intensive case management to support students. The lead Thailand university will provide academic coaches to help the students.

Face to face individual support can be provided via video conferencing using widely available platforms such as MS Office Teams, Zoom, or Skype. On-site visits to ASEAN partner universities would be useful. However, given the cost of travel, site visits would be limited. English is the language of instruction, and Google Translate could be a useful tool to assist students with limited English competency. Translation resources for the four target countries' primary languages are available (Google, n.d.).

It is critical that funding for this program includes resources for the development of nurse educators. Working with the World Health Organization, the World Bank in South Asia, non-governmental agencies, local universities, and national governments Ministry of Health and Education, identifying funding sources to bolster nursing education in the region is achievable. The initial costs would be for a part-time coordinator, nursing faculty, laptops, textbooks, and the internet.

\section{CONCLUSIONS}

Nursing in Thailand has sustained remarkable growth in the past 50 years. The efforts of nursing leaders in Thailand have resulted in significant financial support for nursing education from the Thai government. Progress towards fulfilling the vision of nursing as promoted by Thailand's beloved king and the queen has enabled Thailand to develop nursing expertise on par with nurses in high-income countries. The opportunity for Thailand to build capacity for nursing faculty in the region can help achieve sustainable health goals and universal health coverage. The potential contribution has already begun through collaborations with many low- and middle-income countries. The ability of Thailand to support other ASEAN countries in nursing education is unlimited. Distance education provides a high-quality, low-cost option for ASEAN nurses seeking BNS degrees.

\section{RELEVANCE TO NURSING PRACTICE}

The need for additional nurses throughout ASEAN, particularly Vietnam, Cambodia, Myanmar, and Laos, is urgent. The density of nurses per population in most ASEAN countries is below WHO recommendations (State of the World's Nursing Report -2020, 2020). The traditional method of funding students to move abroad to attend nursing programs is no longer financially viable. Cost-effective distance learning programs provided by nurse educators from the region familiar with the customs, culture, and nursing practice present an opportunity to build nursing capacity. Starting with a pilot bridging BNS program in target countries taught via distance, modalities can demonstrate if such an option is worthwhile.

Given the urgency of meeting the shortage, low- and middle-income countries cannot increase nursing faculty capacity without assistance. Low technology delivery through existing infrastructure with collaborating educational institutions is doable. With laptops preloaded with all textbooks, virtual libraries, eBooks, and faculty access, sound academic programs are feasible. The alternatives to building capacity without high cost are, for most countries, not possible.

Beginning with a BNS bridge program can help provide a cadre of nursing educators in the partnering countries. Program graduates can later go on to become candidates for the master and potentially $\mathrm{Ph} . \mathrm{D}$. or DNP programs. Some Thailand universities have a proven track record of success with nursing education in the region. The need to build nursing capacity is urgent. An innovative approach to nursing education is critical. Distance education provides such an approach.

\section{REFERENCES}

Anders, R. L., \& Kunaviktikul, W. (1999). Nursing in Thailand. Nursing and Health Sciences, 1(4), 235-239.

Cha, C., Hwang, H., An, B., Jeong, S., \& Yang, S. J. (2020). Nursing student and faculty competency improvement through a nurse-bridging program in Cambodia. Nurse education today, 93, 104523 . 
Efendi, F., Nursalam, N., Kurniati, A., \& Gunawan, J. (2018). Nursing qualification and workforce for the association of southeast Asian nations economic community. Nursing Forum, 53(2), 197-203.

Google. (n.d.). Google translates. Retrieved November 15, 2020, from https://translate.google.com/.

International Council of Nurses and nursing now welcome 2020 as international year of the nurse and the midwife. (2020). International Council of Nurses. Retrieved November 15, 2020, from https://www.icn.ch/news/international-council-nurses-and-nursing-now-welcome-2020-international-year-nurse-and-midwife.

Internet penetration in Southeast Asia as of May 2020, by country. (2020). Statista. Retrieved November 15, 2020, from https://www. statista.com/statistics/487965/internet-penetration-in-southeast-asian-countries/.

Kanter, R.M (2008). Men and Women of the Corporation. Basic Books, New York, NY.

Kunaviktikul, W., \& Guptarak, M. (2019). Networking for local and global change: The southeast and east Asian nursing education and research network. Nursing \& Health Sciences, 21(1), $1-3$.

Kunaviktikul, W., Guptarak, M., \& Juntasopeepun, P. (2014). Strengthening nursing education in three southeast Asian countries program (snesea): A model for addressing the nursing shortage on a regional level. Nurse Education Today, 34(5), 683-686.

Kunaviktikul, W., Turale, S., Petrini, M., Tungpunkom, P., \& Avant, K. (2019). Experiences from southeast Asia on nursing education, practice, mobility and influencing policy. International Nursing Review, 66(4), 474-481.

Lall, P., Rees, R., Law, G., Dunleavy, G., Cotič, Ž., \& Car, J. (2019). Influences on the implementation of mobile learning for medical and nursing education: Qualitative systematic review by the digital health education collaboration. Journal of Medical Internet Research, 21(2), e12895.
Liu, Y., Rodcumdee, B., Jiang, P., \& Sha, L. (2015). Nursing education in the United States, Thailand, and China: Literature review. Journal of Nursing Education and Practice, 5(7), Retrieved November 15, 2020, from https://doi.org/10.5430/jnep. v5n7p 100 .

Samuel, L. (2020, June 17). Medical schools are starting to diversify. But they're learning the hard lessons along the way. STAT. Retrieved November 15, 2020, from https://www.statnews. com/2019/06/17/medical-schools-diversity-hard-lessons/.

Shamila, S. (2020, April 7). Countries in WHO South-East Asia Region need 1.9 million more nurses, midwives to achieve health for all. WHO South-East Asia Office. Retrieved November 15, 2020, from https://www.who.int/southeastasia/ news/ detail/07-04-2020-countries-in-who-south-east-asia-regionneed-1.9-million-more-nurses-midwives-to-achieve-health-forall.

State of the world's nursing report -2020. (2020). World Health Organization. Retrieved November 15, 2020, from https:// www.who.int/publications/i/item/nursing-report-2020.

State of the world's nursing: Cambodia. (2020). World Health Organization. Retrieved November 15, 2020, from https:// apps. who.int/nhwaportal/Sown/Files?name $=$ KHM.

State of the world's nursing: Lao People Democratic Republic. (2020). World Health Organization. Retrieved November 15, 2020, from https://apps.who.int/nhwaportal/Sown/Files?name=LAO.

State of the world's nursing: Myanmar. (2020). World Health Organization. Retrieved November 22, 2020, from https://apps. who.int/nhwaportal/Sown/Files?name $=$ MMR.

State of the world's nursing: Viet Nam. (2020, November 15). World Health Organization. https://apps.who.int/nhwaportal/ Sown $/$ Files name $=$ VNM.

Te, V., Griffiths, R., Law, K., Hill, P. S., \& Annear, P. (2018). The impact of Asian economic integration on health worker mobility: A scoping review of the literature. Health Policy and Planning, 33(8), 957-965. 